\title{
PERIODIC SOLUTIONS OF CONVOLUTION TYPE EQUATIONS WITH MONOTONE NONLINEARITY
}

\author{
S.N. ASKHABOV
}

\begin{abstract}
By the method of monotone operators we establish theorems on global existence and uniqueness, as well as estimats and methods of finding the solutions for various classes of nonlinear convolution type integral equations in the real space of $2 \pi$ periodic functions $L_{p}(-\pi, \pi)$.
\end{abstract}

Keywords: nonlinear convolution type equations, monotone operator, potential operator.

Mathematics Subject Classification: 45G10, 47H05

\section{INTRODUCTION}

Many problems of modern mathematics, physics, mechanics and biology give rise to nonlinear convolution type integral equations. For instance, the general class of servomechanisms (tracking systems) is described by nonlinear integral equation of the form [1]

$$
u(x)+\int_{-\infty}^{\infty} h(x-t) \cdot F[t, u(t)] d t=f(x),
$$

where $f(x)$ is the input signal and $h(x)$ is the system response impulse. Equation (1) arises also in the theory of electric nets (signal transmission via a general electric net) involving nonlinear elements (nonlinear resistor) [2]. As $f(x)=0$, equation (1) describes deterministic models of spatial epidemic distribution or of the distribution of auspicious gene in a population along the line with various nonlinearities in epidemic or genetic models, and it is also employed as a mathematical model for some infectious diseases or as a growing equation for some types of population.

Convolution type integral equations with a power nonlinearity arise in the theory of a liquid infiltration from a cylindrical reservoir into an isotropic porous media [5], 6], for describing the propagation of shock wave in tubes filled by a gas [7], as well as for describing the dynamics of an open $p$-adic string for a scalar tachyon field [8]-[10].

The information on other applications of nonlinear convolution type integral equations can be found in monograph [11].

In the present work, employing a new approach, by the method of monotone operators [12] - 14] we establish global estimates on existence, uniqueness and estimates for solutions to various classes of convolution type nonlinear equations in real spaces of $2 \pi$-periodic functions $L_{p}(-\pi, \pi)$ for all values $p \in(1, \infty)$ (see [16]). Similar results for spaces $L_{p}(-\infty, \infty)$ were proved before in [11] subject to the considered class of equations or only for $p \in(1,2]$, or only for $p \in[2, \infty)$ (the matter is that in accordance with Young inequality [11], the convolution

S.N. Askhabov, Periodic solutions of COnvolution type EQuations with monotone nonlinEARITY.

(C) Askhabov S.N. 2016.

The work is supported by RFBR (grant no. 13-01-00422-a).

Submitted July 5, 2015. 
operator acts from space $L_{p}(-\infty, \infty)$ into the dual space $L_{p^{\prime}}(-\infty, \infty), p^{\prime}=p /(p-1)$, only as $p \in(1,2])$. In the considered here case of space $L_{p}(-\pi, \pi)$, by using Young inequality for $p \in(1,2]$ and the embedding $L_{p}(-\pi, \pi) \subset L_{2}(-\pi, \pi) \subset L_{p^{\prime}}(-\pi, \pi)$ as $p \in[2, \infty)$, we show that the convolution operator acts continuously from space $L_{p}(-\pi, \pi)$ into the dual space $L_{p^{\prime}}(-\pi, \pi)$ for all values of $p \in(1, \infty)$ and it is positive. It allows us to the prove the existence and uniqueness theorems with no additional restrictions for $p$. Moreover, in the case of general monotonous (non-power) nonlinearities of general, by combining Banach-Caccioppoli principle and Browder-Minty principle, we show that the solutions of these equations in space $L_{2}(-\pi, \pi)$ can be found by the method of successive approximations of Picard type (cf. [17]), while in the case of power nonlinearities $u^{p-1}$, by employing the potential monoton operators theory, we prove that the solutions can be found by the method of steepest descent (gradient descent) in spaces $L_{p}(-\pi, \pi)$ for each even $p>2$ (cf. [18]).

For the sake of convenience of citations, we provide basic definitions and auxiliary statements used in the present work following the terminology and notations adopted in monograph [14.

Let $X$ be a real Banach space and $X^{*}$ be its dual space. We denote by $\langle y, x\rangle$ the value of a linear continuous functional $y \in X^{*}$ on an element $x \in X$, while by $\|\cdot\|$ and $\|\cdot\|_{*}$ we denote the norms in $X$ and $X^{*}$, respectively.

Definition 1. Let $u, v \in X$ be arbitrary elements. Operator $A: X \rightarrow X^{*}$ (acting from $X$ into $\left.X^{*}\right)$ is called:

monotonous if $\langle A u-A v, u-v\rangle \geqslant 0$;

strictly monotonous if $\langle A u-A v, u-v\rangle>0$ as $u \neq v$;

strongly monotonous if $\langle A u-A v, u-v\rangle \geqslant m \cdot\|u-v\|^{2}, m>0$;

uniformly monotonous if $\langle A u-A v, u-v\rangle \geqslant \beta(\|u-v\|)$, where $\beta$ is an increasing on $[0, \infty)$ function such that $\beta(0)=0$;

coercive if $\langle A u, u\rangle \geqslant \gamma(\|u\|) \cdot\|u\|$, where $\gamma(s)$ is a real function of a non-negative argument such that $\gamma(s) \rightarrow \infty$ as $s \rightarrow \infty$;

Lipschitz continuous if $\|A u-A v\|_{*} \leqslant M \cdot\|u-v\|, M>0$;

bounded Lipschitz continuous if $\|A u-A v\|_{*} \leqslant \mu(r) \cdot\|u-v\|$, where $\mu$ is an increasing on $[0, \infty)$ function, and $r=\max (\|u\|,\|v\|)$;

semi-continuous if function $s \rightarrow\langle A(u+s \cdot v), w\rangle$ is continuous on $[0,1]$ for each fixed $u, v, w \in$ $X$.

The main theorem in the monotone operators theory (see, for instance, [14]) is the BrowderMinty theorem. It is still true if instead of coercitivity condition we assume that operator $A: X \rightarrow X^{*}$ satisfies the condition: $\lim _{\|u\| \rightarrow \infty} \frac{\langle A u, u\rangle}{\|u\|}=\infty$.

If $A$ is a linear operator, then the definitions of monotonous, strictly monotonous and strongly monotone operator coincide respectively with the definition of positive, strictly positive and strongly positive (positive definite) operator [14].

Let $f: X \rightarrow \mathbb{R}$, where $\mathbb{R}=(-\infty, \infty)$, be an arbitrary (not necessarily linear) functional.

Definition 2. Functional $f: X \rightarrow \mathbb{R}$ is called Gâteaux differential if there exists an operator $A: X \rightarrow X^{*}$ such that for all $u, v \in X$ the identity $\lim _{t \rightarrow 0} \frac{f(u+t \cdot v)-f(u)}{t}=\langle A u, v\rangle$ holds true. At that, operator $A$ is called the gradient of functional $f$ and it is denoted by $A=\operatorname{grad} f$.

Definition 3. Operator $A: X \rightarrow X^{*}$ is called potential if there exists a functional $f: X \rightarrow \mathbb{R}$ such that operator $A$ is its gradient. At that, functional $f$ is called the potential of operator $A$.

Example 1. [14]. Let $X$ be a real reflexive Banach space and $A: X \rightarrow X^{*}$ be a linear bounded symmetric operator, i.e., $\langle A u, v\rangle=\langle u, A v\rangle, \forall u, v \in X$. Then $A$ is a potential operator and its potential is $f(u)=\frac{1}{2}\langle A u, u\rangle$. 


\section{Positivity and Potentiality of CONVOLUtion OPERATOR}

In Lebesgue space $L_{p}(-\pi, \pi), 1<p<\infty$, formed by real $2 \pi$-periodic functions, integral convolution operator is

$$
(H u)(x)=\int_{-\pi}^{\pi} h(x-t) u(t) d t
$$

where kernel $h(x) \in L_{1}(-\pi, \pi)$ is a $2 \pi$-periodic function continued to the segment $[-2 \pi, 2 \pi]$.

To find out the conditions for kernel $h(x)$ ensuring that convolution operator $H$ is positive in space $L_{p}(-\pi, \pi)$, we introduce discrete Fourier transform (image) of a sequence of complex numbers $a=\left\{a_{k}\right\}_{k=-\infty}^{\infty}$ :

$$
a(x)=\sum_{k=-\infty}^{\infty} a_{k} \cdot e^{i k x}, \quad \text { where } a_{k}=\frac{1}{2 \pi} \int_{-\pi}^{\pi} a(x) e^{-i k x} d x .
$$

We shall make use of the following two identities:

the formula for convolution of images [21]:

$$
\int_{-\pi}^{\pi} a(x-t) b(t) d t=2 \pi \sum_{k=-\infty}^{\infty} a_{k} b_{k} e^{i k x}
$$

generalized Parseval identity [22]:

$$
\int_{-\pi}^{\pi} a(x) \overline{b(x)} d x=2 \pi \sum_{k=-\infty}^{\infty} a_{k} \overline{b_{k}}
$$

where $b(x)=\sum_{k=-\infty}^{\infty} b_{k} e^{i k x}, b_{k}=\frac{1}{2 \pi} \int_{-\pi}^{\pi} b(x) e^{-i k x} d x$.

Lemma 1. Assume that $1<p<\infty$ and the conditions

$$
\begin{aligned}
& \begin{cases}h(x) \in L_{p /[2(p-1)]}(-\pi, \pi), & \text { if } 1<p \leqslant 2, \\
h(x) \in L_{1}(-\pi, \pi), & \text { if } 2<p<\infty .\end{cases} \\
& h_{c}(k)=\int_{-\pi}^{\pi} h(t) \cos (k \cdot t) d t \geqslant 0 \quad \text { as } k=0,1,2,3, \ldots
\end{aligned}
$$

hold true. Then convolution operator $H$ acts continuously from space $L_{p}(-\pi, \pi)$ into its dual space $L_{p^{\prime}}(-\pi, \pi), p^{\prime}=p /(p-1)$, is positive and for all $u(x) \in L_{p}(-\pi, \pi)$ the inequalities

$$
\begin{gathered}
\|H u\|_{p^{\prime}} \leqslant c_{p, h} \cdot\|u\|_{p} \\
\langle H u, u\rangle=\int_{-\pi}^{\pi}\left(\int_{-\pi}^{\pi} h(x-t) u(t) d t\right) u(x) d x \geqslant 0
\end{gathered}
$$

hold true, where

$$
c_{p, h}= \begin{cases}2 \pi \cdot\|h\|_{p^{\prime} / 2}, & \text { if } \quad 1<p \leqslant 2, \\ (2 \pi)^{2 / p^{\prime}}\|h\|_{1}, & \text { if } \quad 2<p<\infty .\end{cases}
$$

Proof. Let $1<p \leqslant 2$ and $u(x) \in L_{p}(-\pi, \pi)$ be an arbitrary function. Since $h(x) \in$ $L_{p /[2(p-1)]}(-\pi, \pi)$, Young inequality [23] implies immediately that

$$
\|H u\|_{p^{\prime}} \leqslant 2 \pi\|h\|_{p^{\prime} / 2}\|u\|_{p}, \quad \forall u(x) \in L_{p}(-\pi, \pi), 1<p \leqslant 2 .
$$


Suppose that $2<p<\infty$. Then we have continuous embeddings $L_{p}(-\pi, \pi) \subset L_{2}(-\pi, \pi) \subset$ $L_{p^{\prime}}(-\pi, \pi)$, and by Hölder inequality we have

$$
\begin{array}{ll}
\|u\|_{p^{\prime}} \leqslant(2 \pi)^{(p-2) /(2 p)}\|u\|_{2}, & \forall u(x) \in L_{2}(-\pi, \pi), \\
\|u\|_{2} \leqslant(2 \pi)^{(p-2) /(2 p)}\|u\|_{p}, & \forall u(x) \in L_{p}(-\pi, \pi) .
\end{array}
$$

Employing these inequalities as well as inequality (6) for $p=p^{\prime}=2$, we get

$$
\begin{aligned}
\|H u\|_{p^{\prime}} & \leqslant(2 \pi)^{(p-2) /(2 p)}\|H u\|_{2} \leqslant(2 \pi)^{(p-2) /(2 p)} 2 \pi \cdot\|h\|_{1} \cdot\|u\|_{2} \\
& \leqslant(2 \pi)^{(p-2) /(2 p)} 2 \pi \cdot(2 \pi)^{(p-2) /(2 p)}\|h\|_{1} \cdot\|u\|_{p}=(2 \pi)^{2(p-1) / p}\|h\|_{1} \cdot\|u\|_{p},
\end{aligned}
$$

i.e.,

$$
\|H u\|_{p^{\prime}} \leqslant(2 \pi)^{2 / p^{\prime}}\|h\|_{1} \cdot\|u\|_{p}, \quad \forall u(x) \in L_{p}(-\pi, \pi), 2<p<\infty .
$$

It follows immediately from inequalities (6) and (7) that operator $H$ acts continuously from $L_{p}(-\pi, \pi)$ into $L_{p^{\prime}}(-\pi, \pi)$ for each $p \in(1, \infty)$, and inequality (4) holds true.

Let us prove the positivity of operator $H$. By the formula of the convolution of images we have $(H u)(x)=2 \pi \sum_{k=-\infty}^{\infty} h_{k} \cdot u_{k} \cdot e^{i k x}$, where

$$
h_{k}=\frac{1}{2 \pi} \int_{-\pi}^{\pi} h(x) \cdot e^{-i k x} d x, \quad u_{k}=\frac{1}{2 \pi} \int_{-\pi}^{\pi} u(x) \cdot e^{-i k x} d x
$$

Hence,

$$
2 \pi \cdot h_{k} \cdot u_{k}=\frac{1}{2 \pi} \int_{-\pi}^{\pi}(H u)(x) \cdot e^{-i k x} d x
$$

Therefore, employing generalized Parseval identity and the fact that we deal with real functions $u(x)$, we have

$$
\begin{aligned}
\langle H u, u\rangle & =\int_{-\pi}^{\pi}(H u)(x) \cdot \overline{u(x)} d x=2 \pi \sum_{k=-\infty}^{\infty} 2 \pi \cdot h_{k} \cdot u_{k} \cdot \overline{u_{k}} \\
& =(2 \pi)^{2} \cdot\left(h_{0} \cdot\left|u_{0}\right|^{2}+\sum_{k=-\infty}^{-1} h_{k} \cdot\left|u_{k}\right|^{2}+\sum_{k=1}^{\infty} h_{k} \cdot\left|u_{k}\right|^{2}\right) \\
& =(2 \pi)^{2} \cdot\left(h_{0} \cdot\left|u_{0}\right|^{2}+\sum_{k=1}^{\infty}\left[h_{-k} \cdot\left|u_{-k}\right|^{2}+h_{k} \cdot\left|u_{k}\right|^{2}\right]\right) .
\end{aligned}
$$

Since

$$
\begin{aligned}
& \left|u_{-k}\right|^{2}=u_{-k} \cdot \overline{u_{-k}}=\left(\frac{1}{2 \pi} \int_{-\pi}^{\pi} u(t) \cdot e^{i k t} d t\right) \cdot\left(\frac{1}{2 \pi} \int_{-\pi}^{\pi} u(t) \cdot e^{-i k t} d t\right)=\overline{u_{k}} \cdot u_{k}=\left|u_{k}\right|^{2}, \\
& h_{k}+h_{-k}=\frac{1}{2 \pi} \int_{-\pi}^{\pi} h(t)\left[e^{i k t}+e^{-i k t}\right] d t=\frac{1}{\pi} \int_{-\pi}^{\pi} h(t) \cos (k t) d t=\frac{1}{\pi} h_{c}(k),
\end{aligned}
$$


by identity (8) we obtain

$$
\begin{aligned}
\langle H u, u\rangle & =(2 \pi)^{2}\left(\frac{1}{2 \pi} \cdot h_{c}(0) \cdot\left|u_{0}\right|^{2}+\sum_{k=1}^{\infty} \frac{1}{\pi} h_{c}(k) \cdot\left|u_{k}\right|^{2}\right) \\
& =2 \pi \cdot h_{c}(0) \cdot\left|u_{0}\right|^{2}+4 \pi \sum_{k=1}^{\infty} h_{c}(k) \cdot\left|u_{k}\right|^{2} .
\end{aligned}
$$

In view of formula $(9)$ we see that convolution operator $H$ is positive if $h_{c}(k) \geqslant 0$, i.e., if condition (3) holds true.

In the same way one can prove the following lemma dual to Lemma 1.

Lemma 2. Assume that $1<p<\infty$,

$$
\begin{cases}h(x) \in L_{1}(-\pi, \pi), & \text { if } 1<p \leqslant 2 \\ h(x) \in L_{p / 2}(-\pi, \pi), & \text { if } \quad 2<p<\infty\end{cases}
$$

and condition (3) holds true. Then convolution operator $H$ acts continuously from $L_{p^{\prime}}(-\pi, \pi)$ into $L_{p}(-\pi, \pi), p^{\prime}=p /(p-1)$, and is positive, at that,

$$
\|H u\|_{p} \leqslant c_{p, h}^{*} \cdot\|u\|_{p^{\prime}}, \quad \forall u(x) \in L_{p^{\prime}}(-\pi, \pi),
$$

where

$$
c_{p, h}^{*}= \begin{cases}(2 \pi)^{2 / p} \cdot\|h\|_{1}, & \text { if } 1<p \leqslant 2 \\ 2 \pi\|h\|_{p / 2}, & \text { if } 2<p<\infty .\end{cases}
$$

Remark 1. If in Lemmata 1 and 2 we assume additionally that kernel $h(x)$ is an even function, then convolution operator $H$ is potential. Indeed, in the case of an even kernel $h(x)$ operator $H$ is symmetric and, therefore, thanks to Example 1, it is potential.

\section{EXISTENCE AND UNIQUENESS THEOREMS}

Hereafter we assume that given function $F(x, u)$ generating nonlinearity in the considered equation is defined for $x \in[-\pi, \pi], u \in \mathbb{R}$, has period $2 \pi$ w.r.t. $x$ and satisfies Caratheodory conditions [15]: it is measurable in $x$ for each fixed $u$ and is continuous in $u$ for almost each $x$. We denote by $L_{p}^{+}(-\pi, \pi)$ the set of all non-negative functions in $L_{p}(-\pi, \pi)$, while $F$ stands for the superposition operator (Nemytskii operator) generated by function $F(x, u)$.

Theorem 1. Let $1<p<\infty$, kernel $h(x)$ obey conditions (2) and (3), and nonlinearity $F(x, u)$ satisfy the conditions

3.1) $|F(x, u)| \leqslant c(x)+d_{1} \cdot|u|^{p-1}$, where $c(x) \in L_{p^{\prime}}^{+}(-\pi, \pi), d_{1}>0$;

3.2) $F(x, u)$ does not decrease w.r.t. $u$ for almost each $x$;

3.3) $F(x, u) \cdot u \geqslant d_{2} \cdot|u|^{p}-D(x)$, where $D(x) \in L_{1}^{+}(-\pi, \pi), d_{2}>0$, for almost each $x \in[-\pi, \pi]$ and all $u \in \mathbb{R}$. Then for each $\lambda>0$ and $f(x) \in L_{p^{\prime}}(-\pi, \pi)$ equation

$$
\lambda \cdot F[x, u(x)]+\int_{-\pi}^{\pi} h(x-t) u(t) d t=f(x)
$$

has a solution $u^{*}(x) \in L_{p}(-\pi, \pi)$. This solution is unique if in condition 3.2) function $F(x, u)$ strictly increases w.r.t. $u$. Moreover, if condition 3.3) is satisfied as $D(x)=0$, then $\left\|u^{*}\right\|_{p} \leqslant$ $\left(\lambda^{-1} \cdot d_{2}^{-1}\|f\|_{p^{\prime}}\right)^{1 /(p-1)}$. 
Proof. We write equation (11) in operator form: $A u=f$, where $A u=\lambda \cdot F u+H u$. By Lemma 1 and conditions 3.1)-3.3) we obtain that operator $A$ acts continuously from $L_{p}(-\pi, \pi)$ into $L_{p^{\prime}}(-\pi, \pi)$ and is monotonous and coercive. At that, operator $A$ is strictly monotonous if function $F(x, u)$ strictly increases w.r.t. $u$. This is why the statements on the existence and uniqueness of solution are implied by Browder-Minty principle (see, for instance, [14]), which is the main theory of the monotone operators theory. Finally, employing condition 3.3) as $D(x)=0$, the positivity of operator $H$ and the identity $A u^{*}=f$, we have

$$
\begin{aligned}
\lambda \cdot d_{2} \cdot\left\|u^{*}\right\|_{p}^{p} & \leqslant \lambda \cdot\left\langle F u^{*}, u^{*}\right\rangle \leqslant \lambda \cdot\left\langle F u^{*}, u^{*}\right\rangle+\left\langle H u^{*}, u^{*}\right\rangle \\
& =\left\langle A u^{*}, u^{*}\right\rangle=\left\langle f, u^{*}\right\rangle \leqslant\|f\|_{p^{\prime}}\|u\|_{p},
\end{aligned}
$$

that implies immediately the desired estimate for the norm of the solution.

Corollary 1. Let $p \geqslant 2$ be an arbitrary even number, kernel $h(x) \in L_{1}(-\pi, \pi)$ satisfy condition (3). Then equation

$$
u^{p-1}(x)+\int_{-\pi}^{\pi} h(x-t) u(t) d t=f(x)
$$

has the unique solution $u^{*}(x) \in L_{p}(-\pi, \pi)$ for each $f(x) \in L_{p^{\prime}}(-\pi, \pi)$, and $\left\|u^{*}\right\|_{p} \leqslant\|f\|_{p^{\prime}}^{1 /(p-1)}$.

In the next theorem the existence and the uniqueness of the solution to the considered Hammerstein type equation are established without coercitivity of the nonlinearity.

Theorem 2. Let $1<p<\infty, h(x) \in L_{1}(-\pi, \pi)$ as $1<p \leqslant 2$ and $h(x) \in L_{p / 2}(-\pi, \pi)$ as $2<p<\infty$. If kernel $h(x)$ satisfies condition (3) and nonlinearity $F(x, u)$ satisfies condition 3.1) and 3.2) of Theorem 1, then equation

$$
u(x)+\lambda \int_{-\pi}^{\pi} h(x-t) F[t, u(t)] d t=f(x)
$$

has the unique solution $u^{*}(x) \in L_{p}(-\pi, \pi)$ for each $\lambda \geqslant 0$ and each $f(x) \in L_{p}(-\pi, \pi)$. Moreover, if conditions 3.1) and 3.3) hold true as $c(x)=D(x)=0$, then $\left\|u^{*}\right\|_{p} \leqslant d_{1} \cdot d_{2}^{-1} \cdot\|f\|_{p}$.

Proof. As $\lambda=0$, the statement of the theorem is obvious. This is why in what follows we assume that $\lambda>0$. We write equation (12) in operator form: $u+\lambda \cdot H F u=f$. It follows from conditions 3.1) and 3.2) that operator $F$ acts continuously from $L_{p}(-\pi, \pi)$ into $L_{p^{\prime}}(-\pi, \pi)$ and is monotone, while Lemma 2 yields that operator $H$ acts continuously from $L_{p^{\prime}}(-\pi, \pi)$ back to $L_{p}(-\pi, \pi)$ and is positive. Then by Theorem 3 in [19] (see Remark 2 below), this equation has the unique solution $u^{*}(x) \in L_{p}(-\pi, \pi)$.

It remains to prove the estimate for the norm of solution $u^{*}(x)$. Employing conditions 3.1) and 3.3) as $c(x)=D(x)=0$, the positivity of convolution operator $H$ and the identity

$$
u^{*}+\lambda \cdot H F u^{*}=f
$$

we have

$$
d_{2}\left\|u^{*}\right\|_{p}^{p} \leqslant\left\langle u^{*}, F u^{*}\right\rangle+\lambda\left\langle H F u^{*}, F u^{*}\right\rangle=\left\langle f, F u^{*}\right\rangle \leqslant\|f\|_{p}\left\|F u^{*}\right\|_{p^{\prime}} \leqslant d_{1}\|f\|_{p}\left\|u^{*}\right\|_{p}^{p-1},
$$

that implies immediately the desired estimate.

Corollary 2. Let $p \geqslant 2$ be an arbitrary even number, kernel $h(x) \in L_{p / 2}(-\pi, \pi)$ satisfies condition (3). Then the equation

$$
u(x)+\int_{-\pi}^{\pi} h(x-t) u^{p-1}(t) d t=f(x)
$$


has the unique solution $u^{*}(x) \in L_{p}(-\pi, \pi)$ for each $f(x) \in L_{p}(-\pi, \pi)$, and $\left\|u^{*}\right\|_{p} \leqslant\|f\|_{p}$.

Remark 2. The proof of the existence and uniqueness in Theorem 2 is based on Theorem 3 in [19]. It is important to stress that in Theorem 3 concerning Hammerstein equation

$$
u(x)+\int_{a}^{b} K(x, s) F[s, u(s)] d s=f(x),
$$

nonlinearity $F(x, u)$ should not decrease w.r.t. $u$ (it was assumed in [19] that nonlinearity $F(x, u)$ does not increases w.r.t. u). It follows from work [20] by the same authors, where the proof of Theorem 3 in [19] was provided. Moreover, the equation

$$
u(x)-w(x) \int_{a}^{b} w(s) u^{1 / 3}(s) d s=0
$$

with decreasing nonlinearity $F(s, u)=-u^{1 / 3}$ and degenerated kernel $K(x, s)=w(x) \cdot w(s)$ with $w(x) \in L_{4 / 3}(a, b)$, having two different solutions $u_{1}(x)=0$ and $u_{2}(x)=w(x)\left(\int_{a}^{b} w^{4 / 3}(s) d s\right)^{3 / 2}$ in space $L_{4 / 3}(a, b)$ shows that statement on the uniqueness of the solution in Theorem 3 in [19] fails.

Consider now the integral equation where convolution operator $H$ is involved nonlinearly. In this case, as opposed to Theorems 1 and 2 , for nonlinearity $F(x, u)$ we impose conditions ensuring the action of Nemytskii operator $F$ from dual space $L_{p^{\prime}}(-\pi, \pi)$ into original space $L_{p}(-\pi, \pi)$, in which we seek for the solutions; the conditions should also ensure the continuity, strict monotonicity and coercitivity. An important role in studying such equation is played by the existence, strict monotonicity and the coercitivity of inverse operator $F^{-1}$.

Theorem 3. Let $1<p<\infty$, kernel $h(x)$ satisfy conditions (2) and (3), and nonlinearity $F(x, u)$ satisfy the conditions

3.4) $|F(x, u)| \leqslant g(x)+d_{3}|u|^{1 /(p-1)}$, where $g(x) \in L_{p}^{+}(-\pi, \pi), d_{3}>0$;

3.5) $F(x, u)$ strictly increases w.r.t. $u$ for almost each $x$;

3.6) $F(x, u) \cdot u \geqslant d_{4}|u|^{p /(p-1)}-D(x)$, where $D(x) \in L_{1}^{+}(-\pi, \pi), d_{4}>0$.

for almost each $x \in[-\pi, \pi]$ and all $u \in \mathbb{R}$. Then for all $\lambda \geqslant 0$ and $f(x) \in L_{p}(-\pi, \pi)$ equation

$$
u(x)+\lambda \cdot F\left[x, \int_{-\pi}^{\pi} h(x-t) u(t) d t\right]=f(x)
$$

has the unique solution $u^{*}(x) \in L_{p}(-\pi, \pi)$. Moreover, if $g(x)=D(x)=0$ in conditions 3.4) and 3.6), then the estimate

$$
\left\|u^{*}-f\right\|_{p} \leqslant \lambda \cdot\left[d_{3}^{p} \cdot d_{4}^{-1} \cdot c_{p, h} \cdot\|f\|_{p}\right]^{1 /(p-1)}
$$

holds true, where constant $c_{p, h}$ is defined in (5).

Proof. As $\lambda=0$, the statement of the theorem is obvious. This is why in what follows we assume that $\lambda>0$. By Lemma 1, operator $H$ acts from $L_{p}(-\pi, \pi)$ into $L_{p^{\prime}}(-\pi, \pi)$, is continuous and positive. It follows from conditions 3.4)-3.6) that operator $F$ acts back from $L_{p^{\prime}}(-\pi, \pi)$ into $L_{p}(-\pi, \pi)$, is continuous, strictly monotone and coercive. Therefore, by Lemma 2.1 in [11], operator $F$ has the inverse $F^{-1}$, which acts from $L_{p}(-\pi, \pi)$ into $L_{p^{\prime}}(-\pi, \pi)$, is semi-continuous and strictly monotone, and $\lim _{\|v\|_{p} \rightarrow \infty}\left\langle F^{-1} v, v\right\rangle \cdot\|v\|_{p}^{-1}=\infty$. We write equation (13) in operator 
form: $u+\lambda \cdot F H u=f$. Letting $f-u=\lambda \cdot v$ and applying inverse operator $F^{-1}$ to both sides of the resulting equation, we arrive at the equation

$$
\Phi v=H f, \text { where } \Phi v=F^{-1} v+\lambda \cdot H v .
$$

By the above mentioned properties of operators $F^{-1}$ and $H$, operator $\Phi$ acts from $L_{p}(-\pi, \pi)$ into $L_{p^{\prime}}(-\pi, \pi)$, is semi-continuous and strictly monotone, and

$$
\frac{\langle\Phi v, v\rangle}{\|v\|_{p}} \geqslant \frac{\left\langle F^{-1} v, v\right\rangle}{\|v\|_{p}} \rightarrow \infty \text { as }\|v\|_{p} \rightarrow \infty
$$

Hence, by Browder-Minty theorem, equation (14) has the unique solution $v^{*}(x) \in L_{p}(-\pi, \pi)$. Then equation (13) has the solution $u^{*}=f-\lambda \cdot v^{*} \in L_{p}(-\pi, \pi)$.

Let us show that this solution $u^{*}$ is unique. We assume the opposite, i.e., that equation (13) has two different solutions $u_{1}, u_{2} \in L_{p}(-\pi, \pi)$. Then the identities

$$
u_{1}+\lambda \cdot F H u_{1}=f \text { and } u_{2}+\lambda \cdot F H u_{2}=f
$$

hold true. Deducting the former identity from the latter, by (15) we have

$$
u_{2}-u_{1}+\lambda \cdot F H u_{2}-\lambda \cdot F H u_{1}=0
$$

and hence,

$$
\left\langle u_{2}-u_{1}+\lambda \cdot F H u_{2}-\lambda \cdot F H u_{1}, H u_{2}-H u_{1}\right\rangle=0
$$

or

$$
\left\langle u_{2}-u_{1}, H u_{2}-H u_{1}\right\rangle+\lambda \cdot\left\langle F H u_{2}-F H u_{1}, H u_{2}-H u_{1}\right\rangle=0 .
$$

The latter identity is impossible, since the first term in the left hand side is nonnegative by the positivity of operator $H$, while the second term is strictly positive due to the strict monotonicity of operator $F$ and since $H u_{1} \neq H u_{2}$. Indeed, let us show that $H u_{1} \neq H u_{2}$. If we assume the opposite $H u_{1}=H u_{2}$, then it follows from (15) that $u_{1}+\lambda \cdot F H u_{2}=f$ and $u_{2}+\lambda \cdot F H u_{2}=f$. Deducting the left and right hand sides, we obtain $u_{1}-u_{2}=0$ that contradicts to the fact that $u_{1}$ and $u_{2}$ are different.

It remains to prove the estimate for the norm of the solution. We let $\psi=F^{-1} v^{*}$. Then $F \psi=v^{*}$. Since $F^{-1} v^{*}+\lambda \cdot H v^{*}=H f$, then by Lemma 1 and identities $g(x)=D(x)=0$, we have

$$
\begin{aligned}
d_{4}\|\psi\|_{p^{\prime}}^{p^{\prime}} & \leqslant\langle F \psi, \psi\rangle=\left\langle v^{*}, F^{-1} v^{*}\right\rangle \leqslant\left\langle v^{*}, F^{-1} v^{*}\right\rangle+\lambda\left\langle v^{*}, H v^{*}\right\rangle \\
& =\langle F \psi, H f\rangle \leqslant\|F \psi\|_{p}\|H f\|_{p^{\prime}} \leqslant c_{p, h}\|F \psi\|_{p}\|f\|_{p} \leqslant c_{p, h} d_{3}\|\psi\|_{p^{\prime}}^{p^{\prime}-1}\|f\|_{p} .
\end{aligned}
$$

Therefore,

$$
\|\psi\|_{p^{\prime}} \leqslant d_{3} d_{4}^{-1} c_{p, h}\|f\|_{p} .
$$

Since $\left\|v^{*}\right\|_{p}=\|F \psi\|_{p} \leqslant d_{3} \cdot\|\psi\|_{p^{\prime}}^{p^{\prime}-1}$ and $v^{*}=\lambda^{-1} \cdot\left(f-u^{*}\right)$, then

$$
\left\|f-u^{*}\right\|_{p} \leqslant \lambda d_{3}\|\psi\|_{p^{\prime}}^{1 /(p-1)}
$$

that by inequality $(16)$ we obtain the desired estimate for the norm of the solution.

Corollary 3. Let kernel $h(x) \in L_{2}(-\pi, \pi)$ satisfy condition (3). Then the equation

$$
u(x)+\left(\int_{-\pi}^{\pi} h(x-t) u(t) d t\right)^{3}=f(x)
$$

has the unique solution $u^{*} \in L_{4 / 3}(-\pi, \pi)$ for each $f(x) \in L_{4 / 3}(-\pi, \pi)$, and $\left\|u^{*}\right\|_{p} \leqslant\left(\|h\|_{2}\|f\|_{p}\right)^{3}$.

We note that the estimate for the norms of solutions proven in Theorems 13 imply immediately that under the assumptions of these theorems, homogeneous equation (as $f(x)=0$ ) corresponding to equations (11) - (13) have only the trivial solution $u^{*}(x)=0$. 


\section{Approximate solution. Method of successive approximations}

Theorems 13 contain no information on how to find the solutions of equations (11) $-(13)$. In this section, as $p=2$ and under stricter than in Section 3 conditions for the nonlinearity we prove not only the existence and uniqueness of solutions to the considered nonlinear convolution type integral equations but we also justify a was of finding these solutions via the method of successive approximations of Picard type with no restrictions for the value of scalar parameter $\lambda$. In what follows, as usually, we assume that nonlinearity $F(x, u)$ satisfies Caratheodory conditions (see Section 3).

We shall make us of the following theorem being a corollary of well-known results proven in monograph [14].

Theorem 4. Let $H$ be a real Hilbert space and operator $A$ acts from $H$ into $H$. If there exists constants $m>0$ and $M>0(M>m)$ such that for all $u, v \in H$ the inequalities

$$
\|A u-A v\|_{H} \leqslant M \cdot\|u-v\|_{H}, \quad(A u-A v, u-v) \geqslant m \cdot\|u-v\|_{H}^{2}
$$

hold true, then the operator equation $A u=f$ has the unique solution $u^{*} \in H$ for each $f \in H$. This solution can be found by the method of successive approximations by the formulae:

$$
u_{n}=u_{n-1}-\frac{m}{M^{2}} \cdot\left(A u_{n-1}-f\right), \quad n \in \mathbb{N},
$$

and the following estimate for the error

$$
\left\|u_{n}-u^{*}\right\|_{H} \leqslant \frac{m}{M^{2}} \cdot \frac{\alpha^{n}}{1-\alpha} \cdot\left\|A u_{0}-f\right\|_{H}
$$

holds true, where $\alpha=\sqrt{1-m^{2} \cdot M^{-2}}, u_{0} \in H$ is an arbitrary element.

If, in addition, $A$ is a potential operator, this solution can be found by the method of successive approximations defined by the formulae

$$
u_{n}=u_{n-1}-\frac{2}{M+m} \cdot\left(A u_{n-1}-f\right), \quad n \in \mathbb{N},
$$

and the following estimate for the error holds:

$$
\left\|u_{n}-u^{*}\right\|_{H} \leqslant \frac{2}{M+m} \cdot \frac{\alpha^{n}}{1-\alpha} \cdot\left\|A u_{0}-f\right\|_{H}
$$

where $\alpha=(M-m) /(M+m)$.

Proof. Since operator $A$ satisfies two-sided estimates (17), it follows from Theorem 1.4 in [1] that equation $A u=f$ has the unique solution $u^{*} \in H$ and it can be found by iteration formula (18) with estimate $\sqrt{19}$ for the error. If operator $A$ is also potential, then by Theorem 1.7 in [11] this solution can be found by iteration formula (20) with estimate (21) for the error.

Remark 3. By Cauchy-Schwarz inequality, the inequalities in (17) can be simultaneously true only if $m \leqslant M$. Hence, since as $m<M$

$$
\frac{M-m}{M+m}<\sqrt{\frac{M-m}{M+m}}<\frac{M+m}{M} \cdot \sqrt{\frac{M-m}{M+m}}=\sqrt{1-\frac{m^{2}}{M^{2}}},
$$

successive approximations (20) converge to solution $u^{*}$ much faster as (18), i.e., as $n \rightarrow \infty$, the right hand side in (21) tends to zero faster than in (19).

Theorem 4 can be applied to equation 111 . We have the following theorem.

Theorem 5. Let kernel $h(x) \in L_{1}(-\pi, \pi)$ satisfy assumption (3). If for almost each $x \in$ $[-\pi, \pi]$ and all $u_{1}, u_{2} \in \mathbb{R}$ nonlinearity $F(x, u)$ satisfies the conditions

4.1) there exists a constant $M>0$ such that the inequality

$$
\left|F\left(x, u_{1}\right)-F\left(x, u_{2}\right)\right| \leqslant M \cdot\left|u_{1}-u_{2}\right|
$$


holds true;

4.2) there exists a constant $m>0$ such that the inequality

$$
\left[F\left(x, u_{1}\right)-F\left(x, u_{2}\right)\right] \cdot\left[u_{1}-u_{2}\right] \geqslant m \cdot\left|u_{1}-u_{2}\right|^{2}
$$

holds true;

then for each $\lambda>0$ and each $f(x) \in L_{2}(-\pi, \pi)$ equation (11) has the unique solution $u^{*}(x) \in$ $L_{2}(-\pi, \pi)$. This solution can be found by the method of successive approximations defined by the formula

$$
u_{n}=u_{n-1}-\mu \cdot\left(\lambda \cdot F u_{n-1}+H u_{n-1}-f\right), \quad n \in \mathbb{N},
$$

where $\mu=\lambda \cdot m \cdot\left(\lambda \cdot M+\|h\|_{1}\right)^{-2}$, with the estimate for the error

$$
\left\|u_{n}-u^{*}\right\|_{2} \leqslant \mu \cdot \frac{\alpha^{n}}{1-\alpha} \cdot\left\|\lambda \cdot F u_{0}+H u_{0}-f\right\|_{2}
$$

where $\alpha=\sqrt{1-\lambda^{2} m^{2}\left(\lambda \cdot M+\|h\|_{1}\right)^{-2}}, u_{0}(x) \in L_{2}(-\pi, \pi)$ is the initial approximation (arbitrary function). If, in addition, kernel $h(x)$ is an even function, then solution $u^{*}(x)$ can be found by formula (22), where $\mu=2 /\left[\lambda \cdot(M+m)+\|h\|_{1}\right]$, with estimate for (23) the error, where $\alpha=\left[\lambda \cdot(M-m)+\|h\|_{1}\right] /\left[\lambda \cdot(M+m)+\|h\|_{1}\right]$.

Proof. We write equation (11) in operator form $A u=f$, where $A u=\lambda \cdot F u+H u$. It follows from conditions 4.1) and 4.2) that for all $u(x), v(x) \in L_{2}(-\pi, \pi)$ the inequalities

$$
\|F u-F v\|_{2} \leqslant M \cdot\|u-v\|_{2}, \quad(F u-F v, u-v) \geqslant m \cdot\|u-v\|_{2}^{2},
$$

hold true, respectively, i.e., operator $F: L_{2}(-\pi, \pi) \rightarrow L_{2}(-\pi, \pi)$ is Lipschitz-continuous and strongly monotone.

Employing estimates (24), condition (3) and inequality (4), we have

$$
\begin{aligned}
& \|A u-A v\|_{2} \leqslant \lambda \cdot\|F u-F v\|_{2}+\|H(u-v)\|_{2} \leqslant\left(\lambda \cdot M+\|h\|_{1}\right) \cdot\|u-v\|_{2}, \\
& (A u-A v, u-v)=\lambda \cdot(F u-F v, u-v)+(H(u-v), u-v) \geqslant \lambda \cdot m \cdot\|u-v\|_{2}^{2} .
\end{aligned}
$$

Since operator $A$ satisfies all the assumptions of Theorem 4 , then the equation $A u=f$, and therefore, equation (11), have the unique solution $u^{*}(x) \in L_{2}(-\pi, \pi)$, and this solution can be found by iteration formula (22) with estimate (23) for the error.

If in addition we assume that kernel $h(x)$ is an even function, the convolution operator $H$ is potential. Condition 4.1) yields that superposition operator $F$ is also potential [15]. Hence, operator $A=\lambda \cdot F+H$ satisfies the assumptions of Theorem 4, and in accordance with formula (20) and estimate (21) it yields that in (22) and (23) we can take $\mu=2 /\left[\lambda \cdot(M+m)+\|h\|_{1}\right]$, $\alpha=\left[\lambda \cdot(M-m)+\|h\|_{1}\right] /\left[\lambda \cdot(M+m)+\|h\|_{1}\right]$.

It is important to note (see Remark 3 ) that successive approximations $(22)$ corresponding to an even kernel $h(x)$ converge much faster to solution $u^{*}(x)$.

Consider now nonlinear convolution type integral equations $(12)$ and $(13)$. We can not directly apply Theorem 4 to these equations since, generally speaking, the product of monotone operators is not necessarily monotone. This is why in the case of equations (12) and (13) we can construct successive approximations and estimate the rate of the convergence to the exact solution only in terms of inverse operator $F^{-1}$ for superposition operator $F$.

Theorem 6. Let kernel $h(x) \in L_{1}(-\pi, \pi)$ satisfy condition (3), and nonlinearity $F(x, u)$ satisfy conditions 4.1) and 4.2) of Theorem 5. Then for each $\lambda>0$ and each $f(x) \in L_{2}(-\pi, \pi)$ equation (12) has the unique solution $u^{*}(x) \in L_{2}(-\pi, \pi)$. This solution can be found by the formula $u_{n}=F^{-1} v_{n}, n \in \mathbb{N}$, where $F^{-1}$ is the inverse operator for $F$,

$$
v_{n}=v_{n-1}-\mu \cdot\left(F^{-1} v_{n-1}+\lambda \cdot H v_{n-1}-f\right),
$$


with the estimate for the error:

$$
\left\|u_{n}-u^{*}\right\|_{2} \leqslant \mu \cdot \frac{\alpha^{n}}{1-\alpha} \cdot\left\|F^{-1} v_{0}+\lambda \cdot H v_{0}-f\right\|_{2},
$$

where $\mu=m /\left[M^{2}\left(m^{-1}+\lambda\|h\|_{1}\right)^{2}\right], \alpha=\sqrt{1-m^{4} M^{-4}\left(1+\lambda m\|h\|_{1}\right)^{-2}}, v_{0}(x) \in L_{2}(-\pi, \pi)$ is the initial approximation (arbitrary function). If, in addition, kernel $h(x)$ is an even function, then solution $u^{*}(x)$ can be found by iteration formula (25), where $\mu=2 /\left[m^{-1}+\lambda \cdot\|h\|_{1}+m \cdot M^{-2}\right]$ with estimate (26) for the error, where $\alpha=\left[1+\lambda \cdot\|h\|_{1}-m^{2} \cdot M^{-2}\right] /\left[1+\lambda \cdot\|h\|_{1}+m^{2} \cdot M^{-2}\right]$.

Proof. It follows from conditions 4.1) and 4.2) that Nemytskii operator $F$ acts continuously from $L_{2}(-\pi, \pi)$ into $L_{2}(-\pi, \pi)$ and is strongly monotone and inequalities (24) hold true. This is why by Theorems 1.3 and 1.5 in [11], there exists the inverse operator $F^{-1}$, and as $F$, it is potential. At that, for all $u(x), v(x) \in L_{2}(-\pi, \pi)$ the inequalities

$$
\begin{aligned}
& \left\|F^{-1} u-F^{-1} v\right\|_{2} \leqslant m^{-1} \cdot\|u-v\|_{2}, \\
& \left(F^{-1} u-F^{-1} v, u-v\right) \geqslant m \cdot M^{-2} \cdot\|u-v\|_{2}^{2} .
\end{aligned}
$$

hold true. Consider equation (12). We write it in operator form:

$$
u+\lambda \cdot H F u=f .
$$

It is easy to see that if $v^{*} \in L_{2}(-\pi, \pi)$ solves equation

$$
A v=f, \quad \text { where } \quad A v=F^{-1} v+\lambda \cdot H v,
$$

then $u^{*}=F^{-1} v^{*} \in L_{2}(-\pi, \pi)$ is a solution to equation (29) and these solutions are unique in $L_{2}(-\pi, \pi)$ since $F$ and $F^{-1}$ are strongly monotone operators. Since by inequalities (27), (28), condition (3) and estimate (4) the inequalities

$$
\begin{aligned}
& \|A u-A v\|_{2} \leqslant\left\|F^{-1} u-F^{-1} v\right\|_{2}+\lambda \cdot\|H(u-v)\|_{2} \leqslant\left(m^{-1}+\lambda \cdot\|h\|_{1}\right) \cdot\|u-v\|_{2} \\
& (A u-A v, u-v)=\left(F^{-1} u-F^{-1} v, u-v\right)+\lambda \cdot(H(u-v), u-v) \geqslant m \cdot M^{-2} \cdot\|u-v\|_{2}^{2}
\end{aligned}
$$

hold true for all $u(x), v(x) \in L_{2}(-\pi, \pi)$, then operator $A$ satisfies all the assumptions of Theorem 4. Therefore, equation (30) has the unique solution $v^{*}(x) \in L_{2}(-\pi, \pi)$ and this solution can be found by iteration formula 25 , with the estimate for error

$$
\left\|v_{n}-v^{*}\right\|_{2} \leqslant \frac{m \cdot M^{-2}}{\left(m^{-1}+\lambda \cdot\|h\|_{1}\right)^{2}} \cdot \frac{\alpha^{n}}{1-\alpha} \cdot\left\|F^{-1} v_{0}+\lambda \cdot H v_{0}-f\right\|_{2},
$$

where $\alpha=\sqrt{1-m^{4} \cdot M^{-4} \cdot\left(1+\lambda \cdot m \cdot\|h\|_{1}\right)^{-2}}$. Since by inequality (27)

$$
\left\|u_{n}-u^{*}\right\|_{2}=\left\|F^{-1} v_{n}-F^{-1} v^{*}\right\|_{2} \leqslant m^{-1} \cdot\left\|v_{n}-v^{*}\right\|_{2}
$$

by (33) we obtain easily desired estimate (26).

If, in addition, kernel $h(x)$ is an even function, then convolution operator $H$ is potential. Hence, operator $A$ satisfies all the assumptions of Theorem 4, which by formula (20) and estimate (21) yield that in (25) and (26) we can take $\mu=2 /\left[m^{-1}+\lambda \cdot\|h\|_{1}+m \cdot M^{-2}\right]$ and $\alpha=\left[1+\lambda \cdot\|h\|_{1}-m^{2} \cdot M^{-2}\right] /\left[1+\lambda \cdot\|h\|_{1}+m^{2} \cdot M^{-2}\right]$.

We finally prove the following theorem.

Theorem 7. Let kernel $h(x) \in L_{1}(-\pi, \pi)$ satisfy condition (3) and nonlinearity $F(x, u)$ satisfy conditions 4.1) and 4.2) of Theorem 5. Then for each $\lambda>0$ and each $f(x) \in L_{2}(-\pi, \pi)$ equation (13) has the unique solution $u^{*}(x) \in L_{2}(-\pi, \pi)$. This solution can be found by the iteration formula:

$$
u_{n}=u_{n-1}+\lambda \cdot \mu \cdot\left(F^{-1}\left(\lambda^{-1}\left(f-u_{n-1}\right)\right)-H u_{n-1}\right)
$$


where $\mu=m \cdot M^{-2} /\left(m^{-1}+\lambda \cdot\|h\|_{1}\right)^{2}$, with the estimate for the error

$$
\left\|u_{n}-u^{*}\right\|_{2} \leqslant \lambda \cdot \mu \cdot \frac{\alpha^{n}}{1-\alpha} \cdot\left\|F^{-1}\left(\lambda^{-1}\left(f-u_{0}\right)\right)-H u_{0}\right\|_{2},
$$

where $\alpha=\sqrt{1-m^{4} \cdot M^{-4} \cdot\left(1+\lambda \cdot m \cdot\|h\|_{1}\right)^{-2}}, u_{0}(x) \in L_{2}(-\pi, \pi)$ is the initial approximation (arbitrary function). If, in addition, kernel $h(x)$ is an even function, then solution $u^{*}(x)$ can be found by iteration formula (34), where $\mu=2 /\left[m^{-1}+\lambda \cdot\|h\|_{1}+m \cdot M^{-2}\right]$, with estimate (35) for the error, where $\alpha=\left[1+\lambda \cdot\|h\|_{1}-m^{2} \cdot M^{-2}\right] /\left[1+\lambda \cdot\|h\|_{1}+m^{2} \cdot M^{-2}\right]$.

Proof. It follows from conditions 4.1) and 4.2) that (see the proof of Theorem 6) superposition operator $F$ has inverse operator $F^{-1}$ and both the operators are potential and inequalities (24), (27), (28) are satisfied. Consider equation (13). We write it in operator form:

$$
u+\lambda \cdot F H u=f .
$$

It is easy to see that if $v^{*} \in L_{2}(-\pi, \pi)$ solves the equation

$$
F^{-1} v+\lambda \cdot H v=H,
$$

then $u^{*}=f-\lambda \cdot v^{*} \in L_{2}(-\pi, \pi)$ is a solution to equation (36), i.e., to equation (13).

Observing that equation (37) is of the same form as equation (30) (with $H f$ instead of $f$ ), we obtain (see the proof of Theorem 6) that equation (37) has the unique solution $v^{*} \in L_{2}(-\pi, \pi)$ and this solution can be found by iteration formula (25)

$$
v_{n}=v_{n-1}-\frac{m \cdot M^{-2}}{\left(m^{-1}+\lambda \cdot\|h\|_{1}\right)^{2}} \cdot\left(F^{-1} v_{n-1}+\lambda \cdot H v_{n-1}-H f\right),
$$

with estimate for error similar to $(33)$ :

$$
\left\|v_{n}-v^{*}\right\|_{2} \leqslant \frac{m \cdot M^{-2}}{\left(m^{-1}+\lambda \cdot\|h\|_{1}\right)^{2}} \cdot \frac{\alpha^{n}}{1-\alpha} \cdot\left\|F^{-1} v_{0}+\lambda \cdot H v_{0}-H f\right\|_{2},
$$

where $\alpha=\sqrt{1-m^{4} \cdot M^{-4} \cdot\left(1+\lambda \cdot m \cdot\|h\|_{1}\right)^{-2}}$.

By (38) and (39), multiplying by $\lambda$ and taking into consideration that $\lambda \cdot v^{*}=f-u^{*}$ and $\lambda \cdot v_{n-1}=f-u_{n-1}$, we obtain easily formula (34) and estimate (35).

If kernel $h(x)$ is an even function, by the mentioned relation between equations (37) and (30), it follows obviously from the proof of Theorem 6 that in (34) and (35) we can take

$$
\mu=\frac{2}{m^{-1}+\lambda \cdot\|h\|_{1}+m \cdot M^{-2}} \quad \text { and } \quad \alpha=\frac{1+\lambda \cdot\|h\|_{1}-m^{2} \cdot M^{-2}}{1+\lambda \cdot\|h\|_{1}+m^{2} \cdot M^{-2}} .
$$

\section{Approximate solution. Gradient method}

In Section 4 we have considered issues related to approximate solution of convolution type equations (11)-(13) involving general nonlinearities in Lebesgue spaces as $p=2$. The employed methods happen to be inappropriate as $p \neq 2$ since in this case we can not combine contracting mappings principle, which requires an operator to map a given space into itself, with BrowderMinty principle, which requires the operator to map a given space into the dual one. In this section we shall show that if we restrict ourselves by convolution type equations with odd-power nonlinearity of the form $u^{p-1}$, such equations can be approximately solved in Lebesgue space $L_{p}(-\pi, \pi)$ for even $p>2$. At that, as opposed to Section 4 , we employ one of the methods of potential monotone operators theory known as steepest descent method or gradient method.

Definition 4. Banach space $X$ is called strictly convex if for all $u, v \in X$ it follows from $u \neq v,\|u\| \leqslant 1,\|v\| \leqslant 1$ that $\|u+v\|<2$. 
Definition 5. Operator $J: X \rightarrow X^{*}$, where $X^{*}$ is a strictly convex space, is called a dualizing mapping if for each $u \in X$ the identities $\langle J u, u\rangle=\|u\|^{2}=\|J u\|_{*}^{2}$ hold true.

We note that the property of strict convexity of dual space $X^{*}$ in Definition 5 ensures 13 . the uniqueness of dualizing mapping $J: X \rightarrow X^{*}$, and $J$ is a potential operator with potential $f(u)=\frac{1}{2}\|u\|^{2}[14$.

We shall make use of the following theorem implied by known results proven in monograph 14.

Theorem 8. Let $X$ be a real reflexive Banach space and $A: X \rightarrow X^{*}$ be a semi-continuous monotone coercive operator. Then equation $A u=f$ has the unique solution $u^{*} \in X$ for each $f \in X^{*}$. Moreover, if $X$ and $X^{*}$ are strictly convex spaces and operator $A$ is potential and boundedly Lipschitz-continuous, then the sequence $u_{n+1}=u_{n}-\delta_{n} \cdot J^{*}\left(A u_{n}-f\right)$, where $\delta_{n}=\min \left\{1,2 /\left[\varepsilon+\mu\left(\left\|u_{n}\right\|+\left\|A u_{n}-f\right\|_{*}\right)\right]\right\}, n=0,1,2,3, \ldots, J^{*}: X^{*} \rightarrow X$ is a dualizing mapping for $X^{*}, \varepsilon>0$ is an arbitrary number, converges to $u^{*}$ in the sense of the norm in space $X$.

Proof. The existence and uniqueness of solution $u^{*}$ is implied by Browder-Minty principle and the strong convergence of sequence $\left\{u_{n}\right\}$ to $u^{*}$ is implied via mentioned scheme from [14, Thm. 4.2] and [14, Rem. 4.13], since each uniformly monotone operator is strictly monotone and possesses (S)-property [14].

The mentioned in Theorem 8 way of approximate finding solution $u^{*}$ is known as the steepest descent method (or gradient method) [14] since $J^{*} v=\|v\|_{*} \cdot \operatorname{grad}\|v\|_{*}$ for all $v \in X^{*}$ ).

As opposed to Theorem 4, Theorem 8 is applicable to convolution type integral equations with power nonlinearities. Namely, the following theorem holds true and it is in agreement with Corollary 1.

Theorem 9. Let $\alpha=r / s \in[1, \infty)$, where $r, s=1,3,5, \ldots$ are odd number, $f(x) \in$ $L_{1+1 / \alpha}(-\pi, \pi), h(x) \in L_{1}(-\pi, \pi)$ and condition (3) holds true. Then equation

$$
u^{\alpha}(x)+\int_{-\pi}^{\pi} h(x-t) u(t) d t=f(x)
$$

has the unique solution $u^{*}(x) \in L_{1+\alpha}(-\pi, \pi)$. If, in addition, kernel $h(x)$ is an even function and $\alpha>1$ is an odd number, then this solution can be found via successive approximations method by the formula:

$$
u_{n+1}=u_{n}-\delta_{n} \cdot\left\|A u_{n}-f\right\|_{1+1 / \alpha}^{1-1 / \alpha} \cdot\left|A u_{n}-f\right|^{-1+1 / \alpha} \cdot\left[A u_{n}-f\right],
$$

where $n=0,1,2,3, \ldots, u_{0}(x) \in L_{1+\alpha}(-\pi, \pi)$ is an arbitrary function (initial approximation), $A u=u^{\alpha}+H u$,

$$
\delta_{n}=\min \left(1, \frac{2}{\varepsilon+\alpha \cdot\left(\left\|u_{n}\right\|_{1+\alpha}+\left\|A u_{n}-f\right\|_{1+1 / \alpha}\right)^{\alpha-1}+\gamma \cdot\|h\|_{1}}\right),
$$

$\varepsilon>0$ is an arbitrary number, $\gamma=(2 \pi)^{2 \alpha /(\alpha+1)}$.

Proof. We write equation 40 in an operator form

$$
A u=f, \quad \text { where } \quad A u=u^{\alpha}+H u .
$$

The existence and uniqueness of solution $u^{*}(x) \in L_{1+\alpha}(-\pi, \pi)$ of equation 43 is implied by Theorem 1, where we take $p=1+\alpha, \lambda=1, F(x, u)=u^{\alpha}$ and $\alpha=r / s$.

It remains to prove the main statement of the theorem that sequence (41) converges to $u^{*}(x)$ in the sense of the norm in space $L_{1+\alpha}(-\pi, \pi)$. In order to do it, we employ Theorem 8 . 
By Lemma 1, as $p=\alpha+1 \geqslant 2$, we obtain that convolution operator $H$ acts continuously from $L_{1+\alpha}(-\pi, \pi)$ into $L_{1+1 / \alpha}(-\pi, \pi)$ and

$$
\|H u\|_{1+1 / \alpha} \leqslant \gamma \cdot\|h\|_{1} \cdot\|u\|_{1+\alpha}, \quad \text { where } \quad \gamma=(2 \pi)^{2 \alpha /(\alpha+1)} .
$$

Since $u^{\alpha}(x) \in L_{1+1 / \alpha}(-\pi, \pi)$, operator $A$ also acts from $L_{1+\alpha}(-\pi, \pi)$ into $L_{1+1 / \alpha}(-\pi, \pi)$. Let us show that operator $A$ is boundedly Lipschitz-continuous. For each $u, v \in L_{1+\alpha}(-\pi, \pi)$ we have

$$
\|A u-A v\|_{1+1 / \alpha} \leqslant\left\|u^{\alpha}-v^{\alpha}\right\|_{1+1 / \alpha}+\|H(u-v)\|_{1+1 / \alpha}=I_{1}+I_{2} .
$$

Since $\left|t^{\alpha}-s^{\alpha}\right| \leqslant(\alpha / 2) \cdot|t-s| \cdot\left(t^{\alpha-1}+s^{\alpha-1}\right)$ for all $t, s \in \mathbb{R}$ and odd $\alpha \geqslant 3$, then

$$
\begin{aligned}
I_{1} & \leqslant \frac{\alpha}{2}\left(\int_{-\pi}^{\pi}|u(x)-v(x)|^{1+1 / \alpha}\left|u^{\alpha-1}(x)+v^{\alpha-1}(x)\right|^{1+1 / \alpha} d x\right)^{\alpha /(\alpha+1)} \\
& \leqslant \frac{\alpha}{2}\|u-v\|_{1+\alpha}\left(\int_{-\pi}^{\pi}\left|u^{\alpha-1}(x)+v^{\alpha-1}(x)\right|^{(\alpha+1) /(\alpha-1)} d x\right)^{(\alpha-1) /(\alpha+1)} \\
& \leqslant \frac{\alpha}{2}\|u-v\|_{1+\alpha}\left(\|u\|_{1+\alpha}^{\alpha-1}+\|v\|_{1+\alpha}^{\alpha-1}\right) \leqslant \alpha \cdot r^{\alpha-1} \cdot\|u-v\|_{1+\alpha},
\end{aligned}
$$

where $r=\max \left(\|u\|_{1+\alpha},\|v\|_{1+\alpha}\right)$. Thus, estimating $I_{2}$ by inequality (44), we have

$$
\|A u-A v\|_{1+1 / \alpha} \leqslant \mu(r) \cdot\|u-v\|_{1+\alpha},
$$

where $\mu(r)=\alpha \cdot r^{\alpha-1}+\gamma \cdot\|h\|_{1}$ is an increasing on $[0, \infty)$ function. Hence, $A$ is a boundedly Lipschitz-continuous operator.

Let us show that $A$ is an uniformly monotone operator. Employing Lemma 1 and inequality $\left(t^{\alpha}-s^{\alpha}\right) \cdot(t-s) \geqslant 2^{1-\alpha}|t-s|^{\alpha+1}$ valid for all $t, s \in \mathbb{R}$ and odd $\alpha \geqslant 3$, we have

$$
\begin{aligned}
\langle A u-A v, u-v\rangle & \geqslant \int_{-\pi}^{\pi}\left[u^{\alpha}(x)-v^{\alpha}(x)\right] \cdot[u(x)-v(x)] d x \\
& \geqslant 2^{1-\alpha} \cdot\|u-v\|_{1+\alpha}^{1+\alpha}=\beta\left(\|u-v\|_{1+\alpha}\right), \quad \forall u, v \in L_{1+\alpha}(-\pi, \pi),
\end{aligned}
$$

where $\beta(s)=2^{1-\alpha} \cdot s^{\alpha+1}$ is a strictly increasing on $[0, \infty)$ function such that $\beta(0)=0$, i.e., $A$ is an uniformly monotone operator.

Since $F u=u^{\alpha}$ and $H$ are potential operators (see [13] and Remark 1), operator $A$ is also potential. We finally observe that since spaces $L_{1+\alpha}(-\pi, \pi)$ and $L_{1+1 / \alpha}(-\pi, \pi)$ are strictly convex and dualizing mapping $J^{*}$ for space $L_{1+1 / \alpha}(-\pi, \pi)$ is of the form [15]:

$$
J^{*} w(\cdot)=\|w\|_{1+1 / \alpha}^{1-1 / \alpha} \cdot|w(\cdot)|^{1 / \alpha-1} \cdot w(\cdot) .
$$

Therefore, due to Theorem 8, sequence (41) converges to $u^{*}(x)$ in the sense of the norm in space $L_{1+\alpha}(-\pi, \pi)$.

\section{BIBLIOGRAPHY}

1. V.E. Beneš. A nonlinear integral equation from the theory of servo-mechanisms // Bell. System. Techn. J. 40:5, 1309-1321 (1961).

2. V.E. Beneš. A nonlinear integral equation in the Marcinkiewicz space $M_{2} / /$ J. Math. Phys. 44:1, 24-35 (1965).

3. O. Diekman. Thresholds and travelling waves for the geographical spread of infection // J. Math. Biol. 6:2, 109-130 (1978).

4. O.Diekman, H.G. Kaper. On the bounded solutions of nonlinear convolutions equation // Nonl. Anal. Theory. Meth. Appl. 2:6, 721-737 (1978). 
5. J. Goncerzevicz, H. Marcinkowska, W. Okrasiński, K. Tabisz. On the percolation of water from a cylindrical reservoir into the surrounding soil // Zast. Mat. 16:2, 249-261 (1978).

6. W. Okrasiński. Nonlinear Volterra equations and physical applications // Extracta Math. 4:2, 51-74 (1989).

7. J.J. Keller. Propagation of simple nonlinear waves in gas filled tubes with friction // Z. Angew. Math. Phys. 32:2, 170-181 (1981).

8. V.S. Vladimirov, Ya.I. Volovich. Nonlinear dynamics equation in p-adic string theory // Teor. Matem. Fiz. 138:3, 355-368 (2004). [Theor. Math. Phys. 138:3, 297-309 (2004).]

9. V.S. Vladimirov. The equation of the p-adic open string for the scalar tachyon field // Izv. RAN. Ser. Matem. 69:3, 55-80 (2005). [Izv. Math. 69:3, 487-512 (2005).]

10. V.S. Vladimirov. On the non-linear equation of a p-adic open string for a scalar field // Uspekhi Matem. Nauk. 60:6, 73-88 (2005). [Russ. Math. Surv. 60:6, 1077-1092 (2005).]

11. S.N. Askhabov. Nonlinear convolution type equations. Fizmatlit, Moscow (2009). (in Russian).

12. R.I. Kachurovskii. Non-linear monotone operators in Banach spaces // Uspekhi Matem. Nauk. 23:2, 121-168 (1968). [Russ. Math. Surv. 23:2, 117-165 (1968).]

13. M.M. Vainberg. Variational method and method of monotone operators in the theory of nonlinear equations. Nauka, Moscow (1972). [A Halsted Press Book, New York; John Wiley \& Sons; London (1973).]

14. H. Gajewski, K. Greger, K. Zacharias. Nichtlineare Operatorgleichungen und Operatordifferentialgleichungen. Akademie-Verlag, Berlin (1974).

15. M.M. Vaŭnberg. Variational methods for the study of nonlinear operators. Gostekhizdat, Moscow (1956). [Holden-Day Series in Math. Phys. Holden-Day San Francisco (1964).]

16. S.N. Askhabov. Nonlinear convolution-type equations in Lebesgue spaces // Matem. Zametki. 97:5, 643-654 (2015). [Math. Notes. 97:5, 659-668 (2015).]

17. S.N. Askhabov, A.L. Dzhabrailov. Approximate solutions of nonlinear convolution type equations on segment // Ufimskij Matem. Zhurn. 5:2, 3-11 (2013). [Ufa Math. J. 5:2, 3-11 (2013).]

18. S.N. Askhabov. Approximate solution of nonlinear equations with weighted potential type operators // Ufimskij Matem. Zhurn. 3:4, 8-13 (2011). [Ufa Math. J. 3:4, 7-12 (2011).]

19. H. Brezis, F.E. Browder. Some new results about Hammerstein equations // Bull. Amer. Math. Soc. 80:3, 567-572 (1974).

20. H. Brezis, F.E. Browder. Nonlinear integral equations and systems of Hammerstein type // Adv. Math. 18:2, 115-147 (1975).

21. F.D. Gakhov, Yu.I. Cherskii. Equations of convolution type. Nauka, Moscow (1978). (in Russia).

22. R.E. Edwards. Fourier series. A modern introduction. Vol. 2.. Graduate Texts in Mathematics. 85. Springer-Verlag, New York. (1982).

23. A. Zygmund. Trigonometric series. Vol. 1. Cambridge Univ. Press, Cambridge (1959).

Sultan Nazhmudinovich Askhabov,

Chechen State University,

Sheripov str. 32,

364907, Grozny, Russia

E-mail: askhabov@yandex.ru 